\title{
Eight Novel Mutations of the ADAR1 Gene in Chinese Patients with Dyschromatosis Symmetrica Hereditaria
}

\author{
Zhuang-li Tang,, Shuang Wang,, Chen Tu, Tian Wang,, Cheng-wen Ma, Yan Liu, \\ Sheng-xiang Xiao, and Xiao-peng Wang ${ }^{1}$
}

\begin{abstract}
Aims: To identify potential novel gene mutations in Chinese patients with dyschromatosis symmetrica hereditaria (DSH).

Methods: We enrolled 8 Chinese patients with familial DSH, 5 Chinese patients with sporadic DSH, and 100 randomly selected healthy individuals in this study. The genome of each participant was extracted from peripheral blood samples. Sanger sequencing of the $A D A R I$ gene was performed after polymerase chain reaction amplifications. Comparisons between the DNA sequences of the affected individuals and the NCBI database were performed. Results: We detected eight novel heterozygous mutations and five previously reported mutations in the ADARI gene in our patients. The novel mutations include c.1934+3A $>\mathrm{G}$, c.2749A $>\mathrm{G}$, c.2311insA, c.3233G $>\mathrm{A}$, c.3019+1G > T, c.2894C >A, c.1202_1205del, and c.2280C $>$ A. These detected novel mutations are predicted to induce two frame-shift mutations, one nonsense mutation, three missense mutations, and two splice-site mutations. Conclusions: The findings of this study expand our knowledge of the range of ADARl gene mutations in DSH and will contribute to identifying correlations between the various DSH phenotypes and genotypes. Furthermore, they may provide insight into the underlying pathogenic mechanism.
\end{abstract}

Keywords: $A D A R l$, dyschromatosis symmetrica hereditaria, gene mutation

\section{Introduction}

D YSCHROMATOSIS SYMMETRICA HEREDITARIA (DSH; MIM: No. 127400) is a pigmentary genodermatosis of autosomal dominant inheritance. It is characterized by the presence of intermingled hyperpigmented and hypopigmented macules located primarily on the dorsal aspects of the extremities. Occasionally freckle-like macules located on the face can appear in patients with DSH. DSH is caused by pathogenic mutations in the ADARl gene (Miyamura et al., 2003). It is thought to be a rare genodermatosis though the exact frequency of this disease is unknown (Hayashi and Suzuki, 2013) since the epidermal symptoms can be easily ignored by patients owing to numerous factors such as skin color and disease severity.

ADAR1 maps to chromosome 1q21.1-21.2 (Miyamura et al., 2003; Zhang et al., 2003), spans up to $40 \mathrm{~kb}$, and contains 15 exons (Wang et al., 1995). It encodes adenosine deaminase acting on RNA (ADAR), which includes an interferon-inducible long-form protein ADAR1p150 and a constitutively expressed short-form protein ADAR1p110 due to alternative splicing (Patterson and Samuel, 1995). ADAR1 has been long recognized as an essential enzyme for normal mammalian development. Recent researches indicate that ADAR1 not only catalyzes the conversion from adenosine to inosine but also participates in gene regulation through editing-independent manners (Song et al., 2016). Till now, several possible correlations between ADAR1 and human diseases have been identified, but the pathogenic mechanism of DSH still remains unexplained.

In this study, we aimed to identify novel mutations in the $A D A R l$ gene of Chinese patients with DSH. It may provide more information for further investigation on the pathogenic mechanism of DSH and the exact role of ADARI in this disease.

\section{Materials and Methods}

\section{Study subjects}

This study enrolled 13 patients who were diagnosed with DSH at our department from October 2015 to July 2017. Of these, eight patients claimed that members of their pedigree also had clinical manifestations of DSH. In addition, this study involved 100 healthy individuals who were randomly selected from the medical staff and medical students at our institution, and their friends. General physical examinations

Departments of ${ }^{1}$ Dermatology and Venereology and ${ }^{2}$ General Surgery, the Second Affiliated Hospital of Xi' an Jiaotong University, Xi'an, Shaanxi, People's Republic of China.

(C) Zhuang-li Tang et al. 2018; Published by Mary Ann Liebert, Inc. This is an Open Access article distributed under the terms of the Creative Commons Attribution License, which permits unrestricted use, distribution, and reproduction in any medium, provided the original work is properly cited. 
and detailed history taking were conducted in all participants to ensure that none of them had any other diseases that would confuse the diagnosis of DSH.

\section{Genetic studies}

The study protocol was approved by Ethical Committee of the Second Affiliated Hospital of Xi' an Jiaotong University. After informed consent, peripheral blood samples were collected from the participants. Primers flanking all the 15 exons and the adjacent introns of $A D A R 1$ were designed using Primer3.0 (Untergasser et al., 2012). Polymerase chain reaction (PCR) assays of the $A D A R 1$ gene were performed on the extracted genome, as described previously (Li et al., 2010). The purified PCR products of each exon and adjacent intron sequences of $A D A R l$ were directly sequenced on the ABI3730XL DNA analyzer (Applied Biosystems, CA). Novel mutations were identified through comparing the sequencing outcomes with the previously published articles and the single nucleotide polymorphism database.

\section{Results}

All the 13 patients in this study are Han nationality from Shaanxi Province, China. They consist of eight male patients and five female patients with age ranging from 7 to 63 years. The median onset time among the patients is 3 years old. Skin lesions consisted of freckle-like macules distributed on the face and intermingled macules distributed mainly on the dorsal aspects of the extremities. Intermingled lesions of hyperpigmented and hypopigmented macules were found on the extensor aspects of knees in one familial patient. All these skin lesions were of irregular shapes and varied sizes. No other symptoms or signs were involved in all the 13 patients.

The detailed personal information, clinical manifestations, onset time of the disease, and demographic information of the enrolled patients are presented in Table 1 and Figure 1, respectively. Sanger sequencing of PCR products revealed eight novel ADAR1 mutations and five recurrent mutations (Fig. 2 and Table 1). The novel mutations include two frameshift mutations (c.1202_1205del, c.2311insA), three missense mutations (c. $2749 \mathrm{~A}>\mathrm{G}$, c. $2894 \mathrm{C}>\mathrm{A}$, and c.3233G $>\mathrm{A}$ ), one nonsense mutation (c.2280C $>\mathrm{A}$ ), and two splice-site mutations (c. $1934+3 \mathrm{~A}>\mathrm{G}$, c. $3019+1 \mathrm{G}>\mathrm{T})$.

\section{Discussion}

ADAR1 consists of six domains including two Z-DNAbinding domains, three dsRNA-binding domains (DRBMs), and one catalytic domain (deaminase) (Herbert and Rich, 2001). In this study, we identified eight novel and five previously reported $A D A R l$ gene mutations in 13 Chinese patients with DSH. Of the 13 ADARl mutations, 4 locate within the DRBMs while 6 mutations locate within the deaminase.

The two Z-DNA-binding motifs and the three DRBMs enhance the efficiency of RNA editing, but are not essential (Herbert et al., 2002). DRBMs and the C-terminal portion of the catalytic domain have been shown to induce mRNA translation independently (Herbert et al., 2002). Hence mutations in either the DRBMs or the catalytic domain (Hou et al., 2007) are likely to have greater effect than that in the two Z-DNA-binding domains.

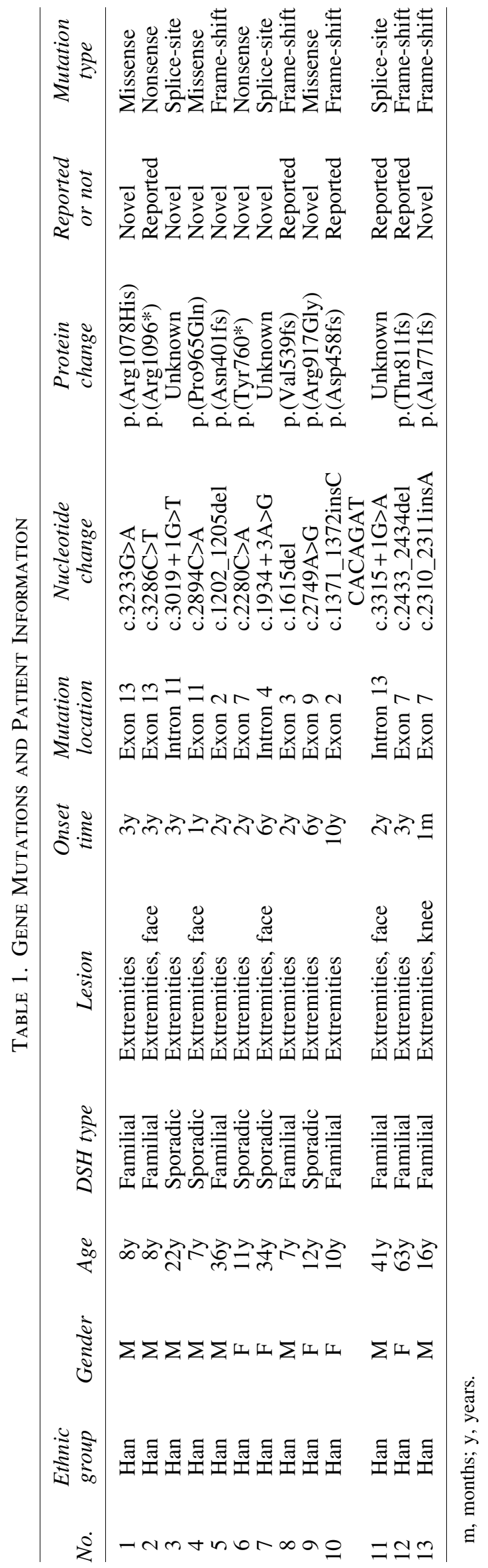



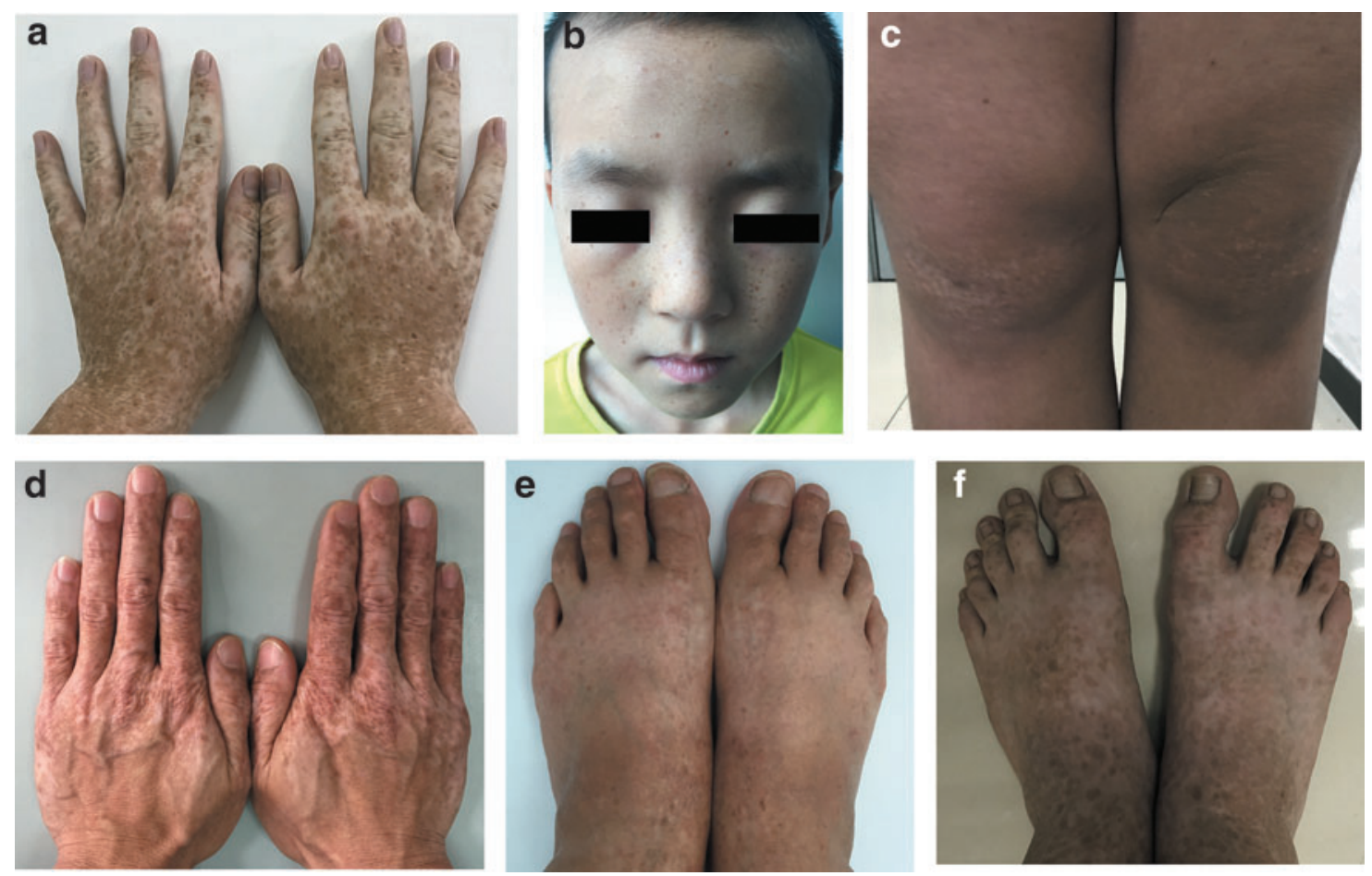

FIG. 1. Clinical manifestations of enrolled patients with dyschromatosis symmetrica hereditaria: (a) clinical manifestations of patient No. 2, intermingled lesions of hyperpigmented and hypopigmented macules on the dorsal aspect of hands; (b) clinical manifestations of patient No. 4, freckle-like macules distributed sparsely on the face; (c) clinical manifestations of patient No. 13, hyperpigmented and hypopigmented macules on the extensor aspect of knees; (d) clinical manifestation of patient No. 5, skin lesions on the hands of a male adult; (e) clinical manifestations of patient No. 11, intermingled lesions of hyperpigmented and hypopigmented macules on the dorsal aspect of feet; (f) clinical manifestations of patient No. 8, skin lesions on the feet of an adolescent male child. Color images available online at www.liebertpub.com/gtmb

Depending on different expression patterns, ADARl encodes two isoforms of proteins due to alternative splicing. The interferon-inducible full-length isoform, ADAR1p150, contains a nuclear export signal at the N-terminal of $\mathrm{Z} \alpha$ domain, thus leading to the main distribution of this protein in the cytoplasm (Poulsen et al., 2001), although the presence of a nuclear localization signal (NLS) in the DRBMs allows it to undergo nucleocytoplasmic shuttling. The shorter isoform, ADAR1p110, consists of merely four domains including the three DRBMs and the catalytic domain, and it is mostly localized in the nucleus owing to NLS (Desterro et al., 2003). It is constitutively expressed and edits dsRNA before nuclear export. It may be more indispensable in maintaining normal life activities (Song et al., 2016).

Mammalian ADAR1 is a type of house-keeping protein and is ubiquitously expressed in almost all organs. The requirement of ADAR1 for life was first recognized from the embryonic lethality of Adarl $^{-1-}$ mice by embryonic day 12.5 (E12.5). Substantial overproduction of interferon and widespread apoptosis were found in these ADARl knockout mice (Wang et al., 2000). Pestal et al. (2015) have demonstrated that ADAR1, typically ADAR1p110, functions as a key regulator of multiorgan development and homeostasis.

Furthermore, ADAR1 is also found indispensable in skin. Sharma created an epidermis-specific $A D A R l$ knockout mouse model to investigate the potential role of $A D A R l$ in skin and the correlation between the gene and the disease. Notably, the mice $\left(\mathrm{ADAR}^{-/}\right.$) undergone such treatment displayed a phenotype of skin that grossly included fur loss, poor skin resiliency, skin rash, and bleeding (Sharma et al., 2011). Interestingly, mice that are heterozygous for $A D A R 1$ deletion $\left(A D A R 1^{+/-}\right)$do not manifest any gross abnormality of the skin even in the extremities and face (Wang et al., 2004) where typical skin lesions of DSH on human sapiens exist.

Up to now, $\sim 180$ mutations in ADARl have been identified worldwide in patients with DSH, whereas most of the cases were reported in East Asia. Mutations in $A D A R 1$ contribute to several hereditary diseases, including DSH, Aicardi-Goutières syndrome (AGS), and bilateral striatal necrosis, although the hereditary pattern may be varied. For decades, most people attributed DSH to haploinsufficiency of ADAR1. Few researches on interferon status of DSH have been conducted (Mannion et al., 2014).

AGS is one hereditary disease of autosomal recessive pattern caused by ADARl mutations (Rice et al., 2012). It is a severe autoimmune disease associated with aberrant interferon response. It has been demonstrated that ADAR1p150 is a unique and specific negative regulator of melanoma differentiationassociated gene 5 (MDA5)—mitochondrial antiviral signaling protein (MAVS) pathway that contributes to AGS (Pestal et al., 2015). MDA5 is a member of the DExD/H box RNA helicase family and it plays a crucial role in antiviral immune response, including producing abundant type-1 interferon (Lee et al., 2015). Song et al. (2016) considered DSH as an autoimmune disease also owing to MDA5-MAVS pathway. Besides, they deemed that ADAR1p150 participated in the pathogenic mechanism of DSH. Whether and how MDA5-MAVS pathway functions in the onset and progression of DSH shall be further investigated. 


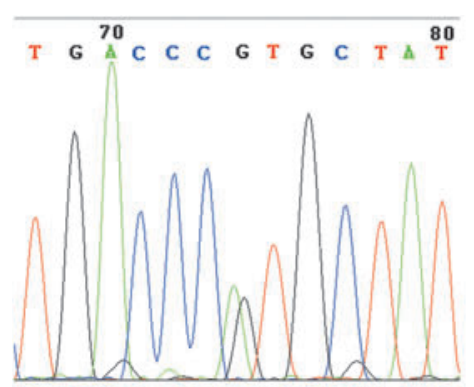

(1)c. $3233 \mathrm{G}>\mathrm{A}$

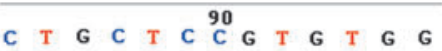

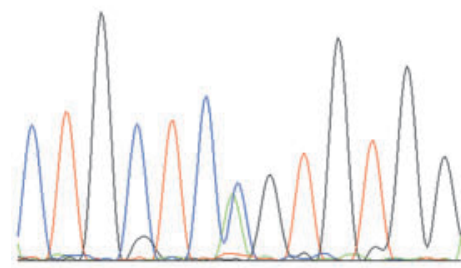

(4)c. $2894 \mathrm{C}>\mathrm{A}$
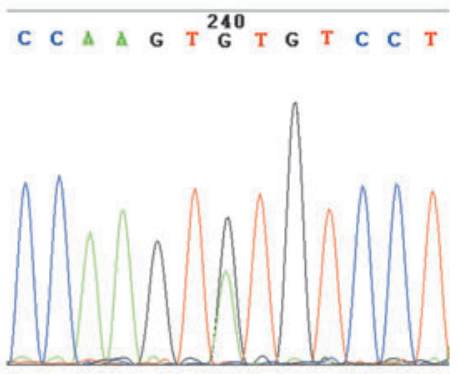

(7)c. $1934+3 A>G$

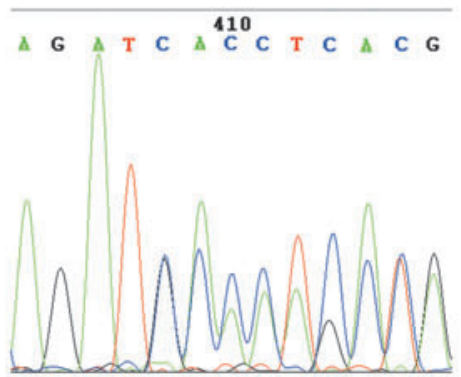

(10)c.1371_1372ins CCACAGAT

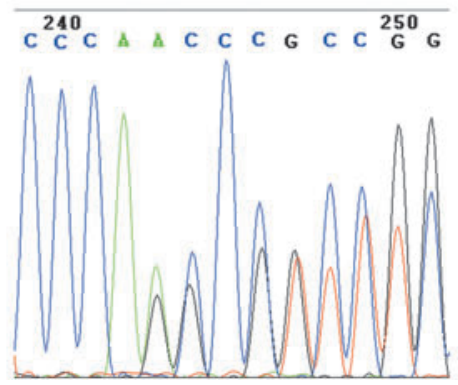

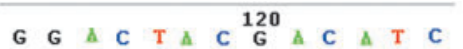

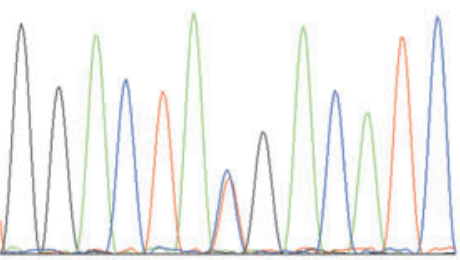

(2)c.3286C $>\mathrm{T}$
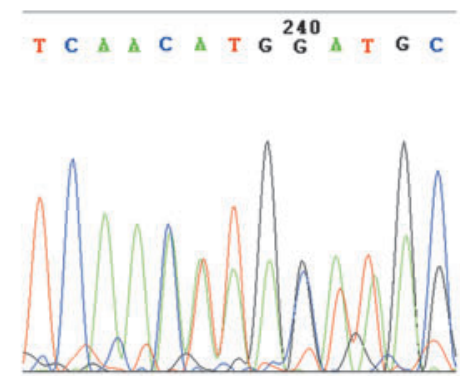

(5)c.1202_1205del
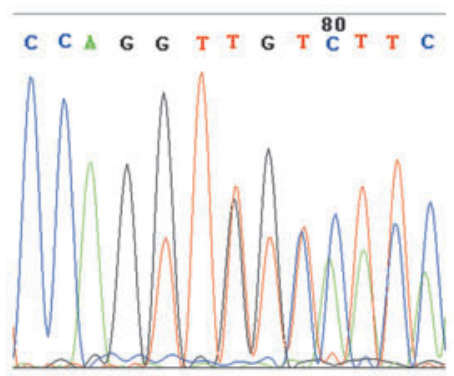

(8)c.1615del

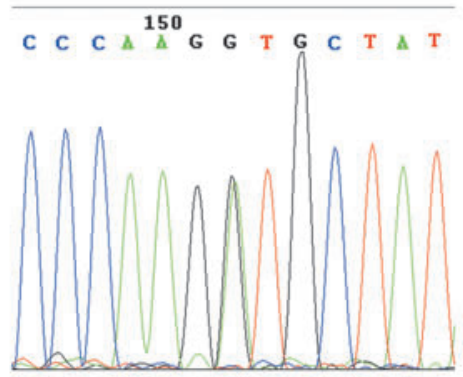

(11)c. $3315+1 \mathrm{G}>\mathrm{A}$ $\triangle G$ A A C G G T ${ }^{210} \triangle{ }^{\circ} G$ T G

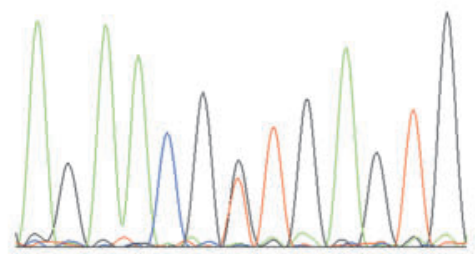

(3)c. $3019+1 \mathrm{G}>\mathrm{T}$
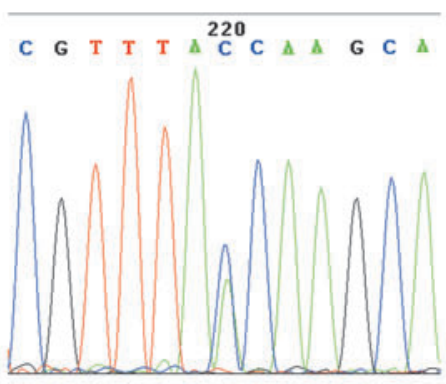

(6)c. $2280 \mathrm{C}>\mathrm{A}$
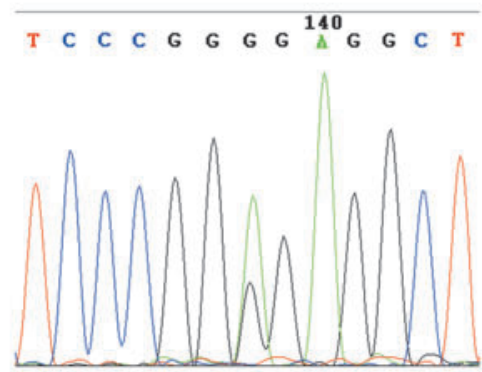

(9)c.2749A $>\mathrm{G}$

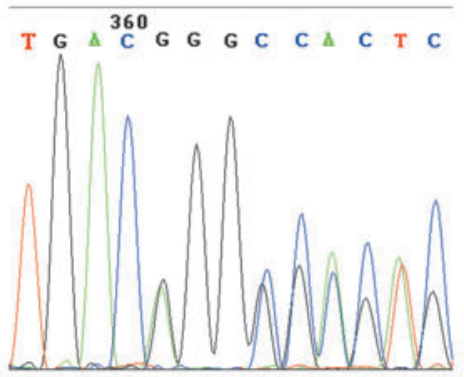

(12)c.2433_2434del

(13)c.2310_2311insA

FIG. 2. Sanger sequencing outcomes of polymerase chain reaction products correspondent to each patient. All the panels are ranged according to the orders of patients listed in Table 1. Color images available online at www.liebertpub.com/gtmb 
Except for its role as a negative regulator in MDA5MAVS pathway, ADAR1 catalyzes adenosine residues to inosine in RNA duplexes, including protein-coding transcripts and numerous noncoding RNAs, including microRNA, siRNA, and lncRNA (Mannion et al., 2014). Editing of endogenous RNA duplexes may decrease the risk of inducing aberrant innate immune response cascades. Furthermore, this may expand the transcriptome when A-to-I editing occurs within coding sequences or at splice sites. Unfortunately, binding and editing of ADAR 1 are generally randomly (Bazak et al., 2014), which may partly account for various phenotypes even in one pedigree.

$A D A R 1$ is typical house-keeping gene and it interacts with other house-keeping genes or tissue-specific genes to perform normal biologic functions (Liu et al., 2014). A hidden crosstalk between $A D A R l$ and unknown tissue-specific genes or proteins may consequently lead to DSH. Further investigations may be conducted based on the assumption.

\section{Conclusion}

In conclusion, we have expanded our knowledge of mutation spectrum in DSH by identifying eight novel ADAR1 mutations. The novel mutations include two frame-shift mutations (c.1202_1205del and c.2311insA), three missense mutations (c.2749A $>\mathrm{G}$, c. $2894 \mathrm{C}>\mathrm{A}$, and c. $3233 \mathrm{G}>\mathrm{A}$ ), one nonsense mutation (c.2280C $>A$ ), and two splice-site mutations (c. $1934+3 \mathrm{~A}>\mathrm{G}$ and c. $3019+1 \mathrm{G}>\mathrm{T})$.

The mechanism of DSH is still mysterious. Involvement of MDA5-MAVS pathway or the interaction between ADAR1 and other tissue-specific genes may account for pathogenic mechanism of DSH. Larger spectrum of ADAR1 mutations will help reveal the essence of the disease and the correlation between phenotypes and genotypes. Further investigations shall be focused on the detailed mechanism and potential signaling pathway.

\section{Acknowledgments}

This study was supported by the Nature Science Foundation of China (grant No. 81101184). The authors appreciate the efforts of the staff members involved in this study and thank the patients for their participation.

\section{Author Disclosure Statement}

No competing financial interests exist.

\section{References}

Bazak L, Haviv A, Barak M, et al. (2014) A-to-I RNA editing occurs at over a hundred million genomic sites, located in a majority of human genes. Genome Res 24:365-376.

Desterro JM, Keegan LP, Lafarga M, et al. (2003) Dynamic association of RNA-editing enzymes with the nucleolus. J Cell Sci 116:1805-1818.

Hayashi M, Suzuki T (2013) Dyschromatosis symmetrica hereditaria. J Dermatol 40:336-343.

Herbert A, Rich A (2001) The role of binding domains for dsRNA and Z-DNA in the in vivo editing of minimal substrates by ADAR1. Proc Natl Acad Sci U S A 98:12132-12137.

Herbert A, Wagner S, Nickerson JA (2002) Induction of protein translation by ADAR1 within living cell nuclei is not dependent on RNA editing. Mol Cell 10:1235-1246.
Hou Y, Chen J, Gao M, et al. (2007) Five novel mutations of RNA-specific adenosine deaminase gene with dyschromatosis symmetrica hereditaria. Acta Derm Venereol 87:18-21.

Lee NR, Kim HI, Choi MS, et al. (2015) Regulation of MDA5MAVS antiviral signaling axis by TRIM25 through TRAF6mediated NF-kappaB activation. Mol Cells 38:759-764.

Li M, Yang L, Li C, et al. (2010) Mutational spectrum of the ADAR1 gene in dyschromatosis symmetrica hereditaria. Arch Dermatol Res 302:469-476.

Liu W, Wang J, Wang T, et al. (2014) Construction and analyses of human large-scale tissue specific networks. PLoS One 9:e115074.

Mannion NM, Greenwood SM, Young R, et al. (2014) The RNA-editing enzyme ADAR1 controls innate immune responses to RNA. Cell Rep 9:1482-1494.

Miyamura Y, Suzuki T, Kono M, et al. (2003) Mutations of the RNA-specific adenosine deaminase gene (DSRAD) are involved in dyschromatosis symmetrica hereditaria. Am J Hum Genet 73:693-699.

Patterson JB, Samuel CE (1995) Expression and regulation by interferon of a double-stranded-RNA-specific adenosine deaminase from human cells: evidence for two forms of the deaminase. Mol Cell Biol 15:5376-5388.

Pestal K, Funk CC, Snyder JM, et al. (2015) Isoforms of RNAediting enzyme ADAR1 independently control nucleic acid sensor MDA5-driven autoimmunity and multi-organ development. Immunity 43:933-944.

Poulsen H, Nilsson J, Damgaard CK, et al. (2001) CRM1 mediates the export of ADAR1 through a nuclear export signal within the Z-DNA binding domain. Mol Cell Biol 21:7862-7871.

Rice GI, Kasher PR, Forte GM, et al. (2012) Mutations in ADAR1 cause Aicardi-Goutieres syndrome associated with a type I interferon signature. Nat Genet 44:1243-1248.

Sharma R, Wang Y, Zhou P, et al. (2011) An essential role of RNA editing enzyme ADAR1 in mouse skin. J Dermatol Sci 64:70-72.

Song C, Sakurai M, Shiromoto Y, et al. (2016) Functions of the RNA editing enzyme ADAR1 and their relevance to human diseases. Genes (Basel) 7:129.

Untergasser A, Cutcutache I, Koressaar T, et al. (2012) Primer3new capabilities and interfaces. Nucleic Acids Res 40:e115.

Wang Q, Khillan J, Gadue P, et al. (2000) Requirement of the RNA editing deaminase ADAR1 gene for embryonic erythropoiesis. Science 290:1765-1768.

Wang Q, Miyakoda M, Yang W, et al. (2004) Stress-induced apoptosis associated with null mutation of ADAR1 RNA editing deaminase gene. J Biol Chem 279:4952-4961.

Wang Y, Zeng Y, Murray JM, et al. (1995) Genomic organization and chromosomal location of the human dsRNA adenosine deaminase gene: the enzyme for glutamate-activated ion channel RNA editing. J Mol Biol 254:184-195.

Zhang XJ, Gao M, Li M, et al. (2003) Identification of a locus for dyschromatosis symmetrica hereditaria at chromosome 1q11-1q21. J Invest Dermatol 120:776-780.

Address correspondence to: Xiao-peng Wang, MD

Department of Dermatology and Venereology

The Second Affiliated Hospital of Xi'an Jiaotong University Xi 5 Road 157 Xi'an 710004 Shaanxi

People's Republic of China

E-mail: wxpdoctor@mail.xjtu.edu.cn 